\title{
Latent class cluster analysis identified hidden headache phenotypes in COVID-19: impact of pulmonary infiltration and IL-6
}

\author{
Ömer Karadaş ${ }^{1,2}$ (D) $\cdot$ Bilgin Öztürk $^{1}$ (D) $\cdot$ Ali Rıza Sonkaya ${ }^{1}$ (D) $\cdot$ Bahar Taşdelen $^{3}$ (D) $\cdot$ Aynur Özge $^{4}$ (D) \\ Hayrunnisa Bolay ${ }^{5}$ (B)
}

Received: 12 October 2020 / Accepted: 7 December 2020 / Published online: 9 February 2021

(C) Fondazione Società Italiana di Neurologia 2021

\begin{abstract}
Background and objective Clinical studies on COVID-19 headache are limited. This prospective study aimed to define headache characteristics, associated clinical and laboratory factors, and treatment response in COVID-19.

Methods Cross-sectional study enrolled 287 patients diagnosed with COVID-19 and hospitalized on a regular ward during the pandemic. All patients were examined face to face and followed by a neurologist during their stay in the hospital. The characteristics, concomitant symptoms, treatment responses, and laboratory findings of COVID-19-associated headaches were recorded.

Results Eighty-three COVID-19 patients reported headache (28.9\%), in which 85.5\% had no prior headaches. Mean age was $48.40 \pm 15.90$ and 58\% was men. Compared to COVID-19 patients without headache $(n=204)$, patients with headache showed significantly higher frequency of pulmonary involvement (76\%) and increased D-dimer levels. Fifty-nine percent of headaches responded iv paracetamol $1000 \mathrm{mg}$, and $85 \%$ of the paracetamol unresponsive headaches were relieved by greater occipital nerve (GON) blocks. Latent class cluster analysis identified 2 distinct class of bilateral, frontal, throbbing headaches: severe (VAS > $84)$, longer ( $>14 \mathrm{~h}$ ), frequent ( $>7$ headache days), paracetamol unresponsive-GON responsive headaches (85\%), with pulmonary involvement $(100 \%)$, and higher IL-6 levels $(>90 \mathrm{pg} / \mathrm{mL})$ were classified in cluster 1 . Cluster 2 included moderately affected patients (VAS $>54,>6 \mathrm{~h},>4$ days, $60 \%$ pulmonary involvement, $>20 \mathrm{pg} / \mathrm{mL}$ IL-6) and paracetamol responsive headaches $(96 \%)$. VAS scores showed positive linear correlation with IL-6 levels $(p<0.001 ; r=0.567)$.

Conclusion The intensity, duration, frequency, bilateral frontal location, and treatment response of COVID-19 headache was related to pulmonary involvement and IL-6 levels, which indicated a role of inflammation in determining the headache manifestations in moderately affected hospitalized patients. ROC curve cutoff values pointed that VAS $>70$ severity, $>9 \mathrm{~h}$ duration, $>$ 5 headache days, and IL- $6>43 \mathrm{pg} / \mathrm{mL}$ levels can be diagnostic for COVID-19 headache. GON blocks can effectively abort headache when patients are unresponsive to paracetamol, and other NSAIDs are avoided during the SARS-CoV-2 infection.
\end{abstract}

Keywords COVID-19 $\cdot$ Headache $\cdot$ Inflammation $\cdot$ IL- $6 \cdot$ Greater occipital nerve

Ömer Karadaș

dromerkaradas@gmail.com

1 Neurology Department, Gülhane School of Medicine, University of Health Science, Ankara, Turkey

2 Neurology Department, Gülhane Training and Research Hospital, Ankara, Turkey

3 Medical Faculty, Department of Biostatistics and Medical Informatics, Mersin University, Mersin, Turkey

4 Medical Faculty, Department of Neurology, Mersin University, Mersin, Turkey

5 Medical Faculty, Department of Neurology, Neuropsychiatry Center, NÖROM Center, Gazi University, Ankara, Turkey

\section{Introduction}

COVID-19 illness, recognized as a pandemic on March 11, 2020 , is caused by the new coronavirus SARS-CoV-2. The disease was defined first with fever, myalgia/exhaustion cough, dyspnea, and involvement the lower respiratory system. Neurotropic and neuroinvasive features of SARS-CoV-2 were also detected in humans with various neurological symptoms including headache, dizziness, smell, and taste impairments $[1-3]$. Other symptoms related to gastrointestinal distress, loss of appetite, and weight loss were also defined during the pandemic $[4,5]$.

Headache symptom was present in approximately $12 \%$ in COVID-19 patients, according to a meta-analysis including 
more than 40,000 patients from 60 studies [6]. Majority of clinical studies generally presented headache rate in their cohort, which was greatly variable depending on the disease severity, recruiting inpatients including ICU or outpatients, obtaining data from hospital records or self-reports/questionnaires [2-7].

Detailed information about headache features associated with COVID-19 was reported in several studies [3, 7-9]. Headache was reported to occur early in the course of the illness and could manifest as an isolated symptom of the disease or presenting symptom of COVID-19 preceding cough or fever $[3,7,8]$. Headache associated with COVID-19 was described as moderate to severe, unrelenting, new-onset, bilateral, frontal pain, and worsening with movements [3, 7-9]. Headache in COVID-19 may be manifestation with migraine-like sensory disturbances such as photophobia, phonohobia, osmophobia, and/or atypical symptoms such as anosmia, or diarrhea, loss of appetite, and weight loss $[3,7,8]$. Recent large survey on 3458 subjects showed that bilateral, long-lasting, analgesicsresistant headaches in men were more frequently associated with COVID-19 infection in conjunction with the anosmia/ ageusia and gastrointestinal complaints [3].

Available reports about the neurological complications of COVID-19 infection are based on retrospective data analysis. To the best of our knowledge, this is the first prospective clinical data acquired by a neurology team serving in a routine pandemic ward. The primary aim of the study is to determine the clinical characteristics of COVID-19 headache and its response to available treatment options. The secondary aim is to evaluate whether there is a relationship between variables of the COVID-19 disease in terms of clinic (comorbid conditions, laboratory findings) and headache features.

\section{Methods}

This cross-sectional clinical study was conducted between March and May 2020, during the pandemic outbreak reached its peak in the country. The study was performed in accordance with the principles of Helsinki Declaration and approved by the Local Ethical Committee (Protocol No: 2020197) and Ministry of Health Ethical Committee (2020-07T1433-58). Written informed consent was obtained from all patients or legal representatives about the recruitment in scientific purposes. All COVID-19 patients were diagnosed and managed by the same treatment protocol approved by the Ministry of Health, consistent with WHO guidelines [10].

Subjects All patients admitted to the emergency department or COVID-19 outpatient clinic were examined, diagnosed, and directed to the appropriate wards or ICU according the severity of the illness. The present study recruited 311 consecutive COVID-19 patients hospitalized with moderate symptoms to the neurology ward, which served as routine COVID-19 ward during the pandemic and managed by a headache specialist.

Inclusion criteria were COVID-19 diagnosis according to WHO interim guidance, adults aged over 18 years, no history of malignancy, severe metabolic syndrome, uncontrolled hypertension, uncontrolled diabetes, chronic liver failure, chronic renal failure, decompensated congestive heart failure, current alcohol/drug abuse and major psychiatric disorders. Exclusion criteria were patients transferred to ICU, headache attributed to other causes, patients who had severe hearing or speech impairment to interfere with communication, and patients who refused further laboratory investigations.

Among 311 patients diagnosed with COVID-19 illness, 287 patients were enrolled in the study. Patients who required mechanical ventilator or ICU within the first 10 days of hospitalization $(n=13)$ and patients diagnosed with other secondary headaches such as sinusitis, meningoencephalitis, and subarachnoid hemorrhage ( $n=11)$ were excluded from the study.

COVID-19 positivity has been defined with the real-time reverse transcription polymerase chain reaction (rRT-PCR) analysis of nasopharyngeal and nasal swab specimens, which is used for SARS-CoV-2 virus analysis. Anamnesis of all patients were taken; also thorax $\mathrm{CT}$ and laboratory tests (complete blood cell count, blood chemical analysis) were investigated in a standardized manner in all patients. D-dimer test has been performed using a fluorescence immunoassay for the quantitative determination of cross-linked fibrin degradation products containing D-dimer in EDTA anticoagulated plasma specimens (reference values $<0.50 \mathrm{mcg} / \mathrm{mL}$ ). IL-6 measurement used $1.5 \mathrm{~mL}$ blood sample in EDTA top tube and processed $0.5 \mathrm{~mL}$ plasma for Quantitative Multiplex Bead Assay (reference values $\leq 1.8 \mathrm{pg} / \mathrm{mL}$ ). Pulmonary involvement was diagnosed by thorax $\mathrm{CT}$, independent of pneumonia symptomatology. Headache specialist and neurologists made faceto-face visits everyday and examined COVID-19 patients. All headache characteristics were questioned carefully and recorded using a semi-structured database. Headache location (bilateral, unilateral, frontal, retroorbital, suboccipital, etc.), headache intensity by visual analog scale (VAS) (VAS 4069 moderate intensity, VAS $>70$ severe intensity), duration of headache attacks per day (hour), and frequency of headache (headache days during the 10 days of hospitalization) were recorded specifically. Hyposmia was diagnosed by examination using two different scents. Diarrhea was diagnosed for who had severe symptoms and prescribed special diet. Loss of weight was defined $>4 \%$ of body weight. Paracetamol $1000 \mathrm{mg}$ was used intravenously as an analgesic and patient with less than $50 \%$ (severity, frequency, or duration) improvement was considered for greater occipital nerve (GON) blocks using $2 \%$ lidocaine $2 \mathrm{~mL}$ per injection points bilaterally.

Statistical analysis In the study, continuous data were summarized as mean \pm standard deviation and median (min-max) 
values, and categorical data were described with count (\%) values. Shapiro-Wilk's test was used to check normality. The comparisons of patients with and without headache were made using independent $t$ test (mean age) or Mann-Whitney $U$ test (median IL-6, D-dimer, VAS, duration of attacks, frequency of attacks). The relationship between categorical variables was evaluated by means of chi-square test and Fisher's exact test. Latent class (LC) cluster analysis, allowing for classification with mixed (categorical and continuous) data, was used to determine the characteristics of the headache associated with COVID-19 and to classify individuals according to their common features. Latent class cluster analysis is a data mining technique and can identify hidden subgroups or clusters exhibiting similar characteristics including clinical features, treatment responses, comorbidities, and outcomes. Local independence assumption of LC cluster analysis was accounted by adding direct effect associated with two variables that had large bivariate residuals $(>2)$, and model fit was checked using Akaike information criteria (AIC) and Bayesian information criteria (BIC) [11]. The validity of the results of cluster analysis was evaluated by comparing the groups in which common features were clustered. To control familywise error rate in these comparisons about main hypothesis of the study, the Bonferroni adjustment was applied. In addition, receiver operating characteristics (ROC) curves were used to obtain cutoff values associated with COVID-19 headache. In contrast to standard statistical techniques, LC cluster models have an advantage that does not require any imputation method for missing values [11]. It is simply based on the density estimation of variables that are observed for the case concerned. In the study, Latent Gold 5.1 and STATISTICA 13.0 packages were used, and statistical significance level was $p<0.05$.

\section{Results}

Among 287 COVID-19 patients, 83 patients reported headache. Demographic features, clinical characteristics, and laboratory results of 2 groups are given in Table 1. Slight male dominance was notable in COVID-19 patients irrespective of headache; age distribution was similar in both groups. In accordance with the ministry of health regulations, all patients received the same standard treatment protocol of hydroxychloroquine, azithromycine, and favipiravir for 5 days and low molecular weight heparin (LMWH) depending on the level of D-dimer elevation. No serious complication was observed except for pulmonary inflammation. There was no mortality, and all patients were discharged after approximately 10 days of hospitalization.

Patients with headache showed higher ratio of pulmonary involvement diagnosed by thorax CT imaging compared to non-headache sufferers $(75.9 \%$ vs $33.8 \%, p<0.001)$. Also, the frequency of comorbid hypertension, diabetes mellitus, and stroke were significantly higher in patients with headache compared to non-headache sufferers (Table 1). The frequency of loss of appetite, weight loss, and diarrhea were higher in patients with headache compared to non-headache group. Loss of appetite lasted up to 15 days, while diarrhea symptom lasted only 3-4 days. Hyposmia, diagnosed by examination, was present in $8.9 \%$ of the COVID-19 patients with headache, while its frequency was $3.6 \%$ in COVID-19 patients without headache. Although mean IL-6 levels were higher in headache patients, the increase was not statistically significant compared to COVID-19 patients without headache. On the other hand, D-dimer levels were significantly higher in headache sufferers compared to those without headache among the COVID-19 patients.

Primary headache frequency was not significantly different in COVID-19 patients with headache (14.5\%) from COVID19 patients without headache $(10.3 \%, p=0.425)$. Eighty-five percent of COVID-19 patients with headache did not have prior primary headaches. There was no significant genderdependent difference in the aspect of headache phenotype. Details of headache characteristics according to gender are shown in Table 2. Fifty-eight percent of COVID-19 patients with headache were men. Previous primary headache disorders were more common in women $(25.7 \%)$ compared to men $(6.3 \%, p=0.013)$. In previous migraine headaches, aura was present in 7 patients; 5 were visual and remaining 2 were somatosensory aura. Previous aura symptoms did not show significant association with headache phenotype, other clinical features, or laboratory data.

Headache during the COVID-19 illness was bilateral, throbbing, or pressing. Most frequent associated symptoms were nausea, photophobia, and phonophobia (Table 2). Most common headache location was frontal (54\%); subsequently occipital (15.6\%); frontotemporal (3.6\%); and rarely parietal, periorbital, and temporal side of the head. Bilateral headache was located frontally compared to other locations $(55.8 \%$ vs $33.3 \%, p=0.011$ ) and more common in patients with pulmonary involvement $(79.2 \%$ vs $20.2 \%, p=0.021)$. Patients with pulmonary involvement reported higher VAS scores compared to the patients without pulmonary involvement (70.71 \pm 16.70 vs $53 \pm 9.09, p<0.001)$.

Compared to the unilateral headaches, bilateral headache location was significantly associated with higher levels of IL$6(55.59 \pm 54.48$ vs $10.07 \pm 7.52, p=0.022)$ and higher levels of D-dimer ( $7.88 \pm 5.57$ vs $3.30 \pm 2.12, p=0.008)$. Scores of VAS showed positive linear correlation with IL-6 levels $(p<0.001 ; r=0.567)$ in COVID-19 patients with headache. Compared to the pressing quality, throbbing quality of headache was associated with a good response to both medical treatment $(61.7 \%$ vs $38.3 \%, p=0.008)$ and interventional management by GON blocks ( $38.3 \%$ vs $0, p<0.001)$. Patients unresponsive to paracetamol regimens reported severe headache 
Table 1 Clinical features and main laboratory findings of patients with COVID-19

\begin{tabular}{llll}
\hline & Patients with headache & Patients without headache & $p$ \\
& $n=83(28.9 \%)$ & $n=204(71.1 \%)$ & \\
\hline Age (mean \pm SD) & $48.40 \pm 15.90$ & $46.05 \pm 14.87$ & 0.236 \\
Median[min-max] & $48.00[20.00-89.00]$ & $46.00[19.00-85.00]$ & \\
Gender (women/men) $n(\%)$ & $35(42.2 \%) / 48(57.8 \%)$ & $92(45.1 \%) / 12(54.9 \%)$ & 0.651 \\
Pulmonary involvement $n(\%)$ & $63(75.9 \%)$ & $69(33.8 \%)$ & $<0.001$ \\
Previous primary HA $n(\%)$ & $12(14.5 \%)$ & $21(10.3 \%)$ & 0.425 \\
Comorbidities & & & \\
Hypertension $n(\%)$ & $33(39.8 \%)$ & $47(23.0 \%)$ & 0.007 \\
DM $n(\%)$ & $14(16.9 \%)$ & $16(7.8 \%)$ & 0.040 \\
CAD $n(\%)$ & $8(9.6 \%)$ & $9(4.4 \%)$ & 0.154 \\
Stroke $n(\%)$ & $9(10.8 \%)$ & $3(1.5 \%)$ & 0.001 \\
Asthma $n(\%)$ & $8(9.6 \%)$ & $10(4.9 \%)$ & 0.218 \\
COPD $n(\%)$ & $9(10.8 \%)$ & $9(4.4 \%)$ & 0.077 \\
Obesity $n(\%)$ & $28(33.7 \%)$ & $49(24.0 \%)$ & 0.124 \\
Hyposmia $n(\%)$ & $7(8.4 \%)$ & $8(3.9 \%)$ & 0.206 \\
Loss of taste $n(\%)$ & $6(7.2 \%)$ & $9(4.4 \%)$ & 0.497 \\
Loss of appetite $n(\%)$ & $45(54.2 \%)$ & $28(13.7 \%)$ & $<0.001$ \\
Loss of weight $\mathrm{n}(\%)$ & $37(44.6 \%)$ & $26(12.7 \%)$ & $<0.001$ \\
Diarrhea $n(\%)$ & $21(25.3 \%)$ & $31(15.2 \%)$ & 0.044 \\
IL-6 (mean $\pm \mathrm{SD})$ & $52.50 \pm 53.85$ & $31.91 \pm 29.01$ & 0.241 \\
Median [min-max] & $26.00[1.00-214.30]$ & $30.70[0.50-114.10]$ & $<0.001$ \\
D-dimer $(\operatorname{mean} \pm \mathrm{SD})$ & $7.55 \pm 5.52$ & $0.80[0.10-5.30]$ & \\
Median[min-max] & $5.52[0.19-42.30]$ & & \\
\hline & & & \\
\hline
\end{tabular}

$H A$ headache, $D M$ diabetes mellitus, $C A D$ coronary artery disease, $C O P D$ chronic obstructive pulmonary disease attacks (VAS scores were $83.97 \pm 6.12$ ) compared to paracetamol responsive group $(54.29 \pm 9.89, \mathrm{p}<0.001)$. Also, COVID19 patients with headache unresponsive to paracetamol had higher levels of IL-6 compared to paracetamol responsive group $(86.34 \pm 59.13$ vs $21.94 \pm 20.66, p<0.001)$. Interestingly patients unresponsive to medical treatment were statistically significantly younger than those responsive patients (median 40 [20-76] vs 52 [25-89], $p=0.033$ ).

Thirty-four out of 83 patients $(40.9 \%)$ were unresponsive to paracetamol. In these patients, interventional GON block procedures were performed for the headache management, and 29 out of 34 patients $(85.2 \%)$ reported pain relief following GON blocks $(p<0.001)$. Patients responsive to GON blocks were younger than unresponsive patients (median 40 [20-71] vs 54 [32-76], $p=0.029)$. GON unresponsive patients $(n=5)$ had a higher IL-6 levels compared to GON responsive patients (median 128.4 [24-214.3] vs 85[4-187], $p<0.001)$.

To determine specific characteristics of COVID-19associated headache, LC cluster analysis was performed. Local dependence assumption of 2-cluster model was checked using bivariate residuals (BVR). Since there was a large BVR $(2.6189>2)$ between location and duration of headache, "direct effect" parameter added to model. After that, all BVRs were less than 1 and model fit was better $(\mathrm{AIC}=895.00$;
$\mathrm{BIC}=774.06)$ than the model which had no direct effect parameter $(\mathrm{AIC}=901.67 ; \mathrm{BIC}=778.31)$.

Patients with headache were clustered into two hidden distinct groups. Headache intensity, frequency and duration of headache attacks, headache location, pulmonary involvement, and IL-6 had an impact on the formation of clusters (Fig. 1). In cluster 1 , headache intensity, frequency, and duration of attacks were statistically significantly higher than the cluster 2 $(p<0.001)$. Remarkably, all cases classified in cluster 1 had pulmonary involvement $(p<0.001)$. These two clusters were also compared in terms of other clinical and laboratory features of the patients. IL- 6 levels in cluster 1 were significantly higher than those in cluster $2(p<0.001)$. Majority of the patients classified in cluster 2 were responsive to paracetamol (94\%), while only 2 out of 33 patients in cluster 1 were responsive to paracetamol $(6 \%)(p<0.001)$. On the other hand, $31 / 34$ patients with GON block procedures were classified in cluster 1 and $78.7 \%$ responded to GON blocks $(p<0.001)$ (Fig. 1).

Also, diagnostic cutoff values of distinguishing two clusters were determined by ROC analysis (Fig. 2). Convincingly, cutoff values calculated by ROC analysis discovered headache intensity of VAS $>70(\mathrm{Se}=1.00,95 \%$ CI: $0.894-1.00$; $\mathrm{Sp}=0.920,95 \% \mathrm{CI}: 0.808-0.978)$, headache frequency of $>5$ 
Table 2 Headache characteristics of the patients with COVID-19 infection

\begin{tabular}{|c|c|c|c|c|}
\hline & $\begin{array}{l}\text { Women } \\
n=35(42 \%)\end{array}$ & $\begin{array}{l}\text { Men } \\
n=48(58 \%)\end{array}$ & $\begin{array}{l}\text { Total } \\
n=83\end{array}$ & $p$ \\
\hline Duration (hours) (mean $\pm \mathrm{SD})$ & $8.69 \pm 4.98$ & $9.23 \pm 5.40$ & $9.00 \pm 5.20$ & 0.728 \\
\hline Median [min-max] & $7.00[3.00-20.00]$ & $7.00[3.00-20.00]$ & $7.00[3.00-20.00]$ & \\
\hline Frequency $($ days $) *($ mean $\pm S D)$ & $4.83 \pm 2.41$ & $5.29 \pm 2.38$ & $5.10 \pm 2.39$ & 0.353 \\
\hline Median [min-max] & $4.00[2.00-9.00]$ & $4.00[2.00-9.00]$ & $4.00[2.00-9.00]$ & \\
\hline Location of headache & & & & 0.693 \\
\hline Unilateral & $3(8.6 \%)$ & $3(6.3 \%)$ & $6(7.2 \%)$ & \\
\hline Bilateral & $32(91.4 \%)$ & $45(93.8 \%)$ & $77(92.8 \%)$ & \\
\hline Quality of headache & & & & 0.077 \\
\hline Throbbing & $20(57.1 \%)$ & $27(56.3 \%)$ & $47(56.6 \%)$ & \\
\hline Pressing & $6(17.1 \%)$ & $15(31.3 \%)$ & $21(25.3 \%)$ & \\
\hline Stabbing & $6(17.1 \%)$ & $1(2.1 \%)$ & $7(8.4 \%)$ & \\
\hline Others & $3(8.6 \%)$ & $5(10.5 \%)$ & $8(9.6 \%)$ & \\
\hline Associated symptoms & & & & 0.841 \\
\hline None & 0 & 0 & 0 & \\
\hline Nausea & $15(42.9 \%)$ & $15(31.3 \%)$ & $30(36.1 \%)$ & \\
\hline Vomiting & $1(2.9 \%)$ & $4(8.3 \%)$ & $5(6.0 \%)$ & \\
\hline Photophobia & $9(25.7 \%)$ & $16(33.3 \%)$ & $25(30.1 \%)$ & \\
\hline Phonophobia & $8(22.8 \%)$ & $15(42.8 \%)$ & $23(27.7 \%)$ & \\
\hline Osmophobia & $1(2.9 \%)$ & $1(2.1 \%)$ & $2(2.4 \%)$ & \\
\hline Aggravation by PA & $5(14.3 \%)$ & $7(14.6 \%)$ & $12(14.5 \%)$ & \\
\hline Vertigo/dizziness & $4(11.4 \%)$ & $5(10.4 \%)$ & $9(10.8 \%)$ & \\
\hline Response to paracetamol $n(\%)$ & $24(68.6 \%)$ & $25(52.1 \%)$ & $49(59.0 \%)$ & 0.200 \\
\hline Response to GON blocks & & & & 0.293 \\
\hline GON-NA & $24(68 \%)$ & $25(52.1 \%)$ & $49(59.0 \%)$ & \\
\hline GON- un responsive & $2(5.7 \%)$ & $3(6.3 \%)$ & $5(6.0 \%)$ & \\
\hline GON- responsive & $9(25.7 \%)$ & $20(41.7 \%)$ & $29(34.9 \%)$ & \\
\hline
\end{tabular}

$N A$ not applicable, $P A$ physical activity, GON great occipital nerve

*Headache days in 10 hospitalization days

headache days $(\mathrm{Se}=0.909,95 \%$ CI: $0.757-0.981 ; \mathrm{Sp}=0.880$, 95\% CI: 0.757-0.955), headache attack duration of $>9 \mathrm{~h}$ $(\mathrm{Se}=0.818,95 \% \mathrm{CI}: 0.645-0.930 ; \mathrm{Sp}=0.920,95 \% \mathrm{CI}$ : $0.808-0.978)$, and IL-6 levels of $>43 \mathrm{pg} / \mathrm{mL}(\mathrm{Se}=0.777$, 95\% CI: 0.577-0.914; $\mathrm{Sp}=0.906,95 \%$ CI: $0.750-0.980$ ). These cutoff values are important for COVID-19-specific headache diagnostic criteria as listed in Fig. 2.

\section{Discussion}

The first cross-sectional study on headache in COVID-19 infection showed that headache symptom was present in $28.9 \%$ of hospitalized patients in routine ward and diagnosed with moderate severity of the illness. During the COVID-19 course, bilateral, frontal, throbbing, severe, long duration, and higher frequency headaches were frequently noticed. The response rate to abortive analgesic medication was limited to $41 \%$, and interventional GON blocks had an impact on headaches unresponsive to paracetamol. Majority of patients had no prior primary headaches. Overall primary headache frequency of $14.5 \%$ in COVID-19 patients was consistent with prevalence of migraine in the population and did not show any increased COVID-19 risk for migraine patients. Patients with headache showed higher frequency of comorbid hypertension, diabetes, and stroke compared to non-headache COVID-19 patients. Notably, COVID-19 patients with headache were associated with increased rate of pulmonary infiltration, increased D-dimer, and IL-6 cytokine levels.

Currently there are no definitive criteria on phenotypic features of COVID-19 headache other than headache disorder attributed to nonspecific viral infection (ICHD-3 code 9.2.2.1) [12]. According to our study new onset, bilateral (particularly in frontal regions), severe, throbbing, long duration headaches accompanied by pulmonary involvement and increased IL-6 levels were suggestive of COVID-19 headache (Fig. 1). These characteristics are in line with proposed specifying features for COVID-19 headache [3, 7-9].

Latent class cluster analysis identified 2 distinct hidden headache phenotypes in COVID-19 patients. Cluster 1 was characterized by severe, long-duration, frequent headache attacks unresponsive to paracetamol $(85 \%)$. Also, this group comprised all patients with pulmonary infiltration and higher levels of IL-6. Cluster 2 classified paracetamol responsive, moderate headaches $(96 \%)$ and patients without pulmonary infiltration (40\%). Headache phenotypes in cluster 1 are in 


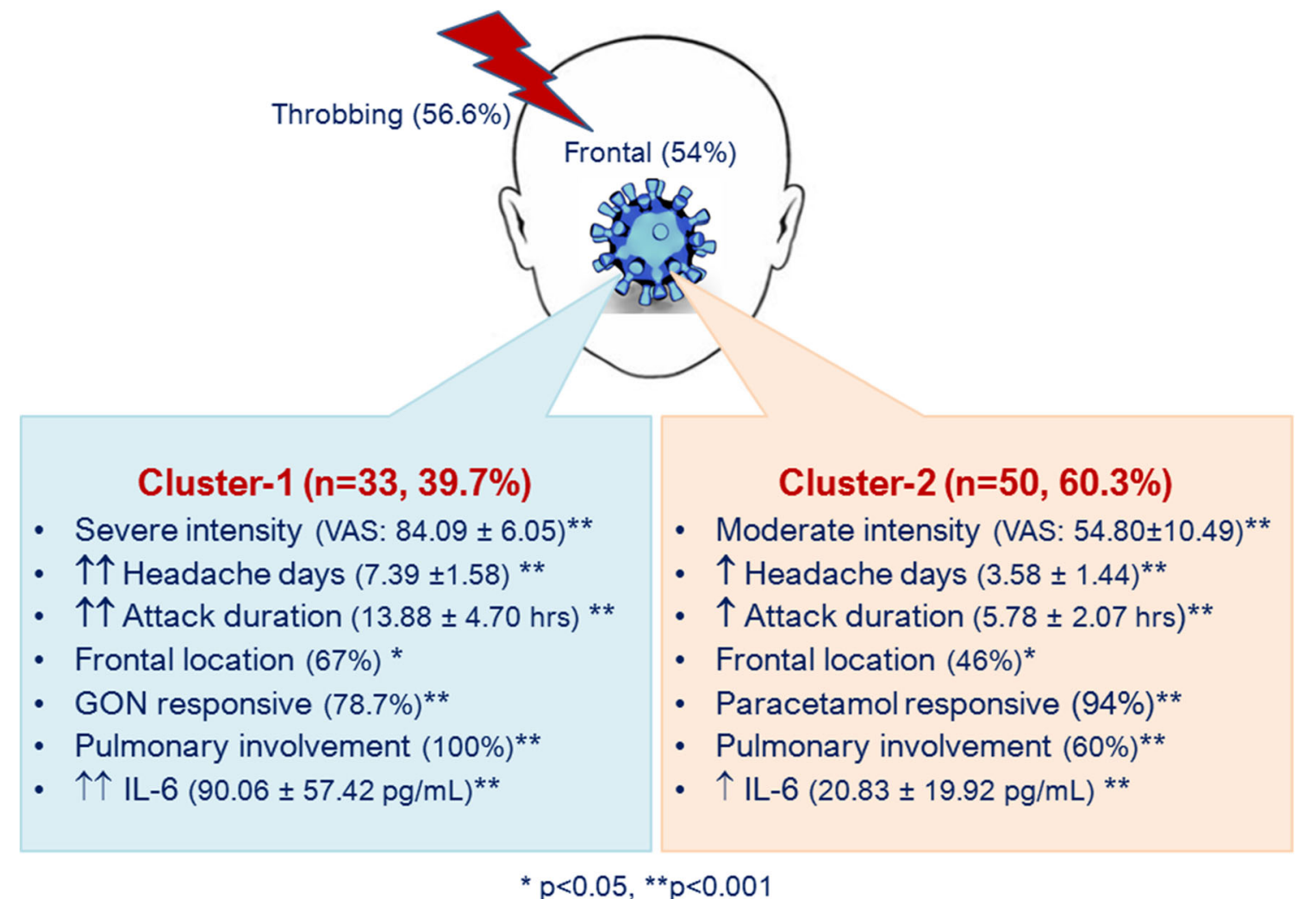

Fig. 1 Latent class cluster analysis identified 2 distinct headache clusters in COVID-19. pulmonary infiltration and circulating proinflammatory IL-6 levels had an impact on the formation of both clusters. Severe headache phenotypes classified in Cluster-1 were blocks. Moderate headache phenotypes were classified in Cluster-2, and $94 \%$ were

accordance with the notion that parenchymal invasion of SARS-CoV-2 is associated with increased circulating cytokines and inflammatory mediators with a potential to progress cytokine storm [13].

Fig. 2 ROC curves for VAS, IL6 , frequency and duration of attacks to classify patients with COVID-19 headache.

(AUROCVAS $=0.980 ; \mathrm{p}<$ 0.001 , AUROCIL- $6=0.867 ; \mathrm{p}<$ 0.001, AUROCFr $=0.940 ; \mathrm{p}<$ 0.001, AUROCD $=0.948 ; * * \mathrm{p}<$ $0.001)$ responsive to paracetamol. Moderate increase of IL-6 and intact pulmonary imaging in $40 \%$ is notable in Cluster-2. Headache in COVID-19 had throbbing quality and was frequently located in the frontal region

Headache symptom denotes a nociceptive process through trigeminal nerve activation and associated with neuroinflammatory-vascular process, where CGRP released from perivascular trigeminal nerve, vascular reactivity, and inflam-

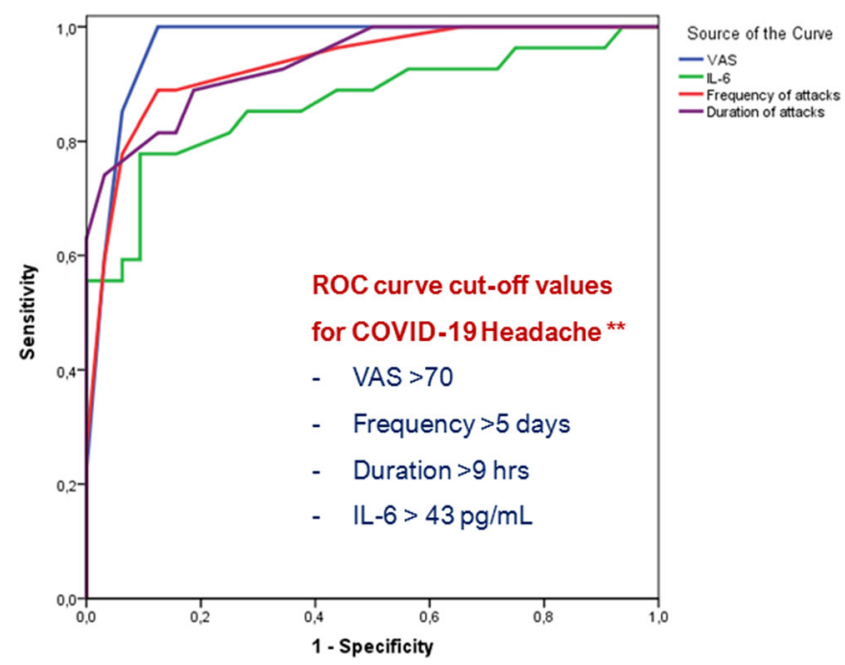


mation take place [14-16]. Several agents such as nitric oxide donors and pro-inflammatory cytokines including IL-6 are well defined triggers for trigemino-vascular system [7, 16]. IL-6, the only cytokine measured in our study, was shown to activate trigeminal afferents in dura mater and induce headache in preclinical models $[17,18]$. Additionally, IL-6 expression in the dural macrophages was induced by nitroglycerin, an established headache trigger $[19,20]$. Also, IL-6 application particularly with heat conditions was shown to provoke CGRP release [21]. Therefore, it is plausible considering the increased CGRP levels as responsible for the headache occurrence. Increased D-dimer is consistent with the altered coagulation related to IL-6 increase in COVID-19 [22]. Higher levels of IL-6 in patients with more intense headache could point out a potential role of IL- 6 in COVID-19 headache. Yet, $40 \%$ of the patients without any pulmonary infiltration in cluster 2 manifested moderate headache intensity and moderate elevation of IL-6. It seems that circulating inflammatory cytokines could also be associated with headache without any pulmonary involvement.

We suppose that the headache correlated with the presence of pulmonary infiltration cannot merely be attributed to hypoxic conditions since severe hypoxemia or hypercapnia was not detected and none of the patients required ventilation. Rather, we propose that pro-inflammatory mediators and cytokines played a crucial role in determining headache manifestations in COVID-19. This proposition was supported by linear positive correlation of IL-6 levels with VAS scores. Also, IL-6 levels had a serious impact on the formation of headache clusters, and ROC curve cutoff values indicated a moderate increase of IL-6 for defining COVID-19 headache (Fig. 2). Recently studies revealed dysregulation of coagulation factors, as a function of circulating IL-6 levels and altered cerebral hemodynamics $[22,23]$, which probably contributed to headaches during COVID-19.

In COVID-19, inflammation takes a central role and SARS-CoV activates NLRP3 inflammasome, which yields pro-inflammatory cytokines including IL-6 [7, 16, 24, 25]. Recently, the possible role of angiotensin system and NLRP3 inflammasome was implicated in the vascular comorbidity and headache development in COVID-19 [16]. Regarding the comorbidity, COVID-19 patients with headache had more frequent hypertension $(39.8 \%$ vs $23.0 \%)$, diabetes ( $16.9 \%$ vs $7.8 \%)$, and stroke ( $10.8 \%$ vs $1.5 \%$ ) compared to non-headache patients. Though COVID-19 patients with cardiovascular comorbidities demonstrated higher mortality in most of the reports $[1,2,26,27]$, no mortality or thromboembolic event was detected, and all patients were discharged in our study group.

Our study samples showed that almost half of the patients (45.9\%) and more than $3 / 4$ of the headache sufferers showed pulmonary involvement detected by thorax CT. Those patients with headache symptoms showed more frequent pulmonary involvement compared to non-headache sufferers (75.9\% vs $33.8 \%)$. Intriguingly, D-dimer levels were significantly higher in patients with headache compared to nonheadache sufferers $(7.55 \pm 5.52$ vs $1.13 \pm 0.99)$. Related to severity of the infection, elevated D-dimer levels and IL-6 were correlated with a risk of thrombosis and poor prognosis $[1,2,26,27]$. All patients received treatment and LMWH from the admission, and we believe that interference was the critical to reduce the risk of thrombosis and related complications in our study group. Besides, relatively younger age (mean $48.40 \pm 15.90 \mathrm{vs} 46.05 \pm 14.87$ ) of the study population had an impact on the outcome. Additionally, presence of headache in hospitalized COVID-19 patients was stated as an independent predictor of lower risk of mortality [28].

Treatment was the same for all patients according to the protocol. Therefore, the headache occurrence cannot be attributed to the treatment difference. The headache was responsive to intravenous $1000 \mathrm{mg}$ paracetamol, yet it was relapsing. Peripheral nerve blocks particularly GON blocks are commonly used procedures in headache clinical practice for the management of several headache types [29, 30]. GON blocks effectively relieved headache in $85 \%$ of paracetamol unresponsive COVID-19 patients in the presented study. Relief with GON procedures may engage secondary mechanisms and achieve long-term benefit decreasing systemic side effects of pharmacologic agents. Consequently, GON blocks seem to be a good choice to abort headache attacks when patients were unresponsive to paracetamol and other NSAIDs are avoided during the SARS-CoV-2 pandemic.

Headache symptom was associated with diarrhea in COVID-19 patients (25\% vs $15 \%$ ). Patients diagnosed with COVID-19 manifested GI distress symptoms including nausea, vomiting, abdominal pain, and diarrhea [3-5, 8], and headache was reported around $22 \%$ in patients manifesting GI symptoms [4]. Yet, the mechanism of GI symptoms and headache association is not clear; CGRP a neuropeptide released from sensory nerve endings could link two symptoms $[7,8,31]$.

Increased rate of loss of appetite and weight was seen in patients with headache compared to non-headache COVID-19 patients $(54.2 \%, 44.6 \%$ vs $13.7 \%, 12.7 \%$ respectively). The loss of weight was disproportionate to the diarrhea rate in patients. Rapid loss of appetite and weight extending beyond the duration diarrhea was also reported in headache associated with mild COVID-19 patients [8]. Our study sample showed low hyposmia rates $(5 \%)$ and good recovery rate in all patients, suggesting that the loss of odor was not a predictor for the outcome of COVID-19 [5].

Our clinical study has some constraints as it was conducted during extraordinary conditions in pandemic outbreak peak. Patient recruitment was dependent on patient management algorithm of the emergency department and infection committee policy regulated general treatment algorithm including requirement of hospitalization in routine wards. Additionally, 
several reports warning to avoid NSAIDs particularly ibuprofen [32] left paracetamol as an only abortive medication for headaches during COVID-19 infection. Another limitation of the study was its restriction to hospitalized patients in general ward without including patients in the ICU and nonhospitalized mild symptomatic patients. Other potential limitation was lack of long-term follow-up data providing temporal course of COVID-19 headaches.

This prospective study on headache in COVID-19 has several implications. Clinical data was obtained from face-to-face examination and daily follow-ups. Study was conducted by a neurology team who were expert in headache area including peripheral nerve interventions. Also, application of latent class cluster analysis revealed novel associations of symptoms and signs clustered around COVID-19 infection headache. The study also included associations between headache features, treatment responses, and laboratory findings in COVID-19. Cutoff values of ROC curves could be used to define COVID-19 infection headache. We showed for the first time that clinical features could be helpful for predicting headache treatment response and GON blocks can be safely used in the management of COVID-19 infection headache.

\section{Conclusion}

Headache symptom in COVID-19 is characterized by new onset, bilateral, frontal, throbbing, severe, long-lasting attacks in patients without prior primary headaches. The intensity, duration, frequency, bilateral frontal location, and treatment response of COVID-19 headache are related to pulmonary involvement and IL-6 levels, indicating a significant role of inflammation and cytokines in determining the headache manifestations in moderately affected hospitalized patients. Latent class cluster analysis identified 2 distinct class of headaches depending on the intensity, duration, frequency, pulmonary involvement, IL-6 levels, and treatment response. ROC curve cutoff values pointed that VAS $>70,>9$ h duration, $>5 / 10$ headache day frequency, and IL- $6>43 \mathrm{pg} / \mathrm{mL}$ can be diagnostic for COVID-19 headache.

\section{Compliance with ethical standards}

Conflict of interest None

Ethical approval None

\section{References}

1. Karadaş Ö, Öztürk B, Sonkaya AR (2020) A prospective clinical study of detailed neurological manifestations in patients with COVID-19. Neurol Sci 41(8):1991-1995. https://doi.org/10.1007/ s10072-020-04547-7
2. Favas TT, Dev P, Chaurasia RN, Chakravarty K, Mishra R, Joshi D, Mishra VN, Kumar A, Singh VK, Pandey M, Pathak A (2020) Neurological manifestations of COVID-19: a systematic review and meta-analysis of proportions. Neurol Sci 41:3437-3470. https://doi.org/10.1007/s10072-020-04801-y

3. Özge Uygun Ö, Ertaş M, Ekizoğlu E, Bolay H, Özge A, Kocasoy E, Çağatay A, Baykan B (under review). https://www.researchsquare. com/article/rs-59636/v1 (accessed 11.01.2021) The emergence of a new form of headache in COVID-19 pandemic era. J Headache and Pain. https://doi.org/10.21203/rs.3.rs-59636/v1

4. Jin X, Lian JS, Hu JH et al (2020) Epidemiological, clinical and virological characteristics of 74 cases of coronavirus-infected disease 2019 (COVID-19) with gastrointestinal symptoms. Gut. 320926

5. Lechien JR, Chiesa Estomba CM, De Siati DR et al (2020) Olfactory and gustatory dysfunctions as a clinical presentation of mild-to-moderate forms of the coronavirus disease (COVID-19): a multicenter European study. Eur Arch Otorhinolaryngol. https:// doi.org/10.1007/s00405-020-05965-1

6. Fu L, Wang B, Yuan T, Chen X, Ao Y, Fitzpatrick T, Li P, Zhou Y, Lin YF, Duan Q, Luo G, Fan S, Lu Y, Feng A, Zhan Y, Liang B, Cai W, Zhang L, du X, Li L, Shu Y, Zou H (2020) Clinical characteristics of coronavirus disease 2019 (COVID-19) in China: a systematic review and meta-analysis. J Infect 80:656-666

7. Bolay H, Gül A, Baykan B (2020) COVID-19 is a real headache! Headache 60:1415-1421

8. Toptan T, Aktan Ç, Başarı A, Bolay H (2020) Case series of headache characteristics in COVID-19: headache can be an isolated symptom [published online ahead of print, 2020 Aug 13]. Headache. https://doi.org/10.1111/head.13940

9. Porta-Etessam J, Matías-Guiu JA, González-García N, et al (2020) Spectrum of headaches associated with SARS-CoV-2 infection: study of healthcare professionals [published online ahead of print, 2020 Jul 15]. Headache. https://doi.org/10.1111/head.13902

10. WHO (2020) Clinical management of severe acute respiratory infection when novel coronavirus (nCoV) infection is suspected. https://www.who.int/publications-detail/clinical-management-ofsevere-acute-respiratoryinfection-when-novelcoronavirus-(ncov)infection-is-suspected. Accessed 20 Nov 2020

11. Vermunt JK, Magidson J (2016) Upgrade manual for Latent GOLD 5.1 Belmont Massachusetts: Statistical Innovations Inc

12. Headache Classification Committee of the International Headache Society (IHS). The international classification of headache disorders, 3rd edition. Cephalalgia. 2018;38:1-211

13. Song P, Li W, Xie J, Hou Y, You C (2020) Cytokine storm induced by SARS-CoV-2. Clin Chim Acta 509:280-287. https://doi.org/10. 1016/j.cca.2020.06.017

14. Ashina M, Hansen JM, Do TP, Melo-Carrillo A, Burstein R, Moskowitz MA (2019) Migraine and the trigeminovascular system-40 years and counting. Lancet Neurol 18(8):795-804

15. Bolay H, Reuter U, Dunn AK, Huang Z, Boas DA, Moskowitz MA (2002 Feb) Intrinsic brain activity triggers trigeminal meningeal afferents in a migraine model. Nat Med 8(2):136-142

16. Bolay H, Ozge A, Uludüz D, Baykan B (2020 Nov) Are migraine patients at increased risk for symptomatic Covid-19 due to shared comorbidities? Headache. 60(10):2508-2521. https://doi.org/10. 1111/head.13998

17. Yan J, Melemedjian OK, Price TJ et al (2012) Sensitization of dural afferents underlies migraine-related behavior following meningeal application of interleukin-6 (IL-6). Mol Pain 8:6

18. Burgos-Vega CC, Quigley LD, Trevisan Dos Santos G et al (2019) Non-invasive dural stimulation in mice: a novel preclinical model of migraine. Cephalalgia. 39(1):123-134

19. Reuter U, Bolay H, Jansen-Olesen I et al (2001) Delayed inflammation in rat meninges: implications for migraine pathophysiology. Brain. 124(Pt 12):2490-2502 
20. Reuter U, Chiarugi A, Bolay H, Moskowitz MA (2002) Nuclear factor-kappa B as a molecular target for migraine therapy. Ann Neurol 51(4):507-516

21. Oprée A, Kress M (2000) Involvement of the proinflammatory cytokines tumor necrosis factor-alpha, IL-1 beta, and IL- 6 but not IL-8 in the development of heat hyperalgesia: effects on heatevoked calcitonin gene-related peptide release from rat skin. J Neurosci 20(16):6289-6293

22. D'Alessandro A, Thomas T, Dzieciatkowska M et al (2020) Serum proteomics in COVID-19 patients: altered coagulation and complement status as a function of IL-6 level [published online ahead of print, 2020 Aug 14]. J Proteome Res. https://doi.org/10.1021/acs. jproteome.0c00365

23. Sonkaya AR, Öztürk B, Karadas Ö (2020 Oct.) Cerebral hemodynamic alterations in patients with Covid-19. Turkish Journal of Medical Sciences. https://doi.org/10.3906/sag-2006-203

24. Shah A (2020) Novel coronavirus-induced NLRP3 inflammasome activation: a potential drug target in the treatment of COVID-19. Front Immunol 11:1021

25. Nieto-Torres JL, Verdiá-Báguena C, Jimenez-Guardeño JM, Regla-Nava JA, Castaño-Rodriguez C, Fernandez-Delgado R, Torres J, Aguilella VM, Enjuanes L (2015) Severe acute respiratory syndrome coronavirus E protein transports calcium ions and activates the NLRP3 inflammasome. Virology. 485:330-339

26. Wang D, Yin Y, Hu C et al (2020) Clinical course and outcome of 107 patients infected with the novel coronavirus, SARS-CoV-2, discharged from two hospitals in Wuhan, China. Critical Care 24(1):188
27. ÖzdağAcarli AN, Samanci B, Ekizoğlu E et al (2020) Coronavirus disease 2019 (COVID-19) from the point of view of neurologists: observation of neurological findings and symptoms during the combat against a pandemic. Noro Psikiyatr Ars 57(2):154-159. Published 2020 May 1. https://doi.org/10.29399/npa.26148

28. Trigo J, García-Azorín D, Planchuelo-Gómez Á et al (2020) Factors associated with the presence of headache in hospitalized COVID19 patients and impact on prognosis: a retrospective cohort study. $\mathrm{J}$ Headache Pain 21(1):94. Published 2020 Jul 29. https://doi.org/10. 1186/s10194-020-01165-8

29. Ozge A, Uludüz D (2019) Introduction to interventional procedures; timing and patient selection. In: Ozge A, Uluduz D, Karadas O, Bolay $\mathrm{H}$ (eds) Peripheral interventional management in headache. Springer, pp 7-16. https://doi.org/10.1007/978-3-03010853-3

30. Karadaş Ö, Özön AÖ, Özçelik F, Özge A (2017) Greater occipital nerve block in the treatment of triptan-overuse headache: a randomized comparative study. Acta Neurol Scand 135(4):426-433

31. Falkenberg K, Bjerg HR, Olesen J (2020) Two-hour CGRP infusion causes gastrointestinal hyperactivity: possible relevance for CGRP antibody treatment. Headache. 60(5):929-937. https://doi. org/10.1111/head.13795

32. Little P (2020) Non-steroidal anti-inflammatory drugs and covid19. BMJ 368:m1185. Published 2020 Mar 27. https://doi.org/10. 1136/bmj.m1185

Publisher's note Springer Nature remains neutral with regard to jurisdictional claims in published maps and institutional affiliations. 